\title{
Review of: "Indirect effects of parental conflict on conspecific offspring development"
}

\author{
David Haig ${ }^{1}$ \\ 1 Harvard University
}

Potential competing interests: The author(s) declared that no potential competing interests exist.

Jenn Coughlan has presented the latest installment in her analysis of the Mimulus guttatus speciescomplex. Coughlan's previous work has shown patterns of hybrid seed inviability in crosses within the complex that are compatible with predictions of 'parental conflict' theory. The novelty of the study is that Coughlan examined effects of mixed pollinations on the size of non-hybrid seeds.

Some background will be helpful.

(1) In species with an initial syncytial stage in endosperm development, a common pattern is observed in reciprocal crosses between diploids and their own autotetraploids. When the diploid is the maternal parent and the tetraploid is the paternal parent, there is an increased number of mitotic divisions before cellularization whereas, in the reciprocal cross, there is a reduced number of mitotic divisions. This pattern is consistent with normal diploid development reflecting a balance between genes of maternal origin 'favoring' fewer divisions and smaller seeds and genes of paternal origin 'favoring' more divisions and larger seeds.

(2) A similar pattern is observed in reciprocal crosses between species at the same ploidy level in which cellularization is delayed in one direction of the cross but accelerated in the other direction of the cross. Yaniv Brandvain and myself proposed that this pattern could be explained by different degrees of outbreeding in the two species. Within each species, normal develop is determined by a balance between antagonistic effects of maternal genes favoring fewer mitotic divisions before cellularization and paternal genes favoring more mitotic divisions. In inbreeding species, the degree of 'parental antagonism' is relatively small compared to that present in outbreeding species. In crosses between species with different mating systems, when outbreeder is the father and the inbreeder is the mother, a 'strong' paternal force is opposed by a 'weak' maternal counter-force resulting in extra mitotic divisions before cellularization. In the reciprocal cross, a 'weak' paternal force is opposed by a 'strong' maternal counter-force resulting in fewer mitotic divisions and smaller seeds. We called this the 'weak inbreeder/strong outbreeder' hypothesis (WISO or 'Why so?'). 
The manuscript presents a study of seed size in crosses involving three populations Mimulus guttatus (MG), 'northern Mimulus decorus' (ND), and 'southern Mimulus decorus' (SD). Coughlan's previous work had shown that these populations could be arranged in order of genomic 'strength' ND < MG < SD. In this study, Coughlan compares sized of non-hybrid seeds in selfed pollinations and mixed pollinations involving the three populations. The prediction was that non-hybrid seeds would be larger from mixed pollinations than selfed pollinations when the non-self pollen came from a population with lower genomic strength but would be smaller when the non-self pollen came from a population with higher genomic strength. The logic is that hybrid seeds would make weaker 'demands' on the mother in crosses of the first kind but stronger 'demands' in crosses of the second kind.

The experiments involved measuring the size of a very large number of seeds and produced results consistent with the theoretical prediction. It should be noted that the effects are relatively weak (increases and decreases of average seed size of a few percent). However, population genetics typically considers fitness differences of a few percent to be strong selection. Coughlan's work stands out for its combination of experimental elegance and deep theoretical understanding. This is an uncommon combination.

Coughlan JM, Brown MW, Willis JH (2020) Patterns of hybrid seed inviability in the Mimulus guttatus sp. complex reveal a potential role of parental conflict in reproductive isolation. Current Biology 30: 83-93.

Brandvain Y, Haig D (2005) Divergent mating systems and parental conflict as a barrier to hybridization in flowering plants. American Naturalist 166: 330-338.

Haig D, Westoby M (1991) Genomic imprinting in endosperm: its effects on seed development in crosses between species and between different ploidies of the same species, and its implications for the evolution of apomixis. Philosophical Transactions of the Royal Society B 333: 1-13. 